\title{
Digital panoramic radiography for diagnosis of the temporomandibular joint: CBCT as the gold standard
}

\section{Daniela Brait Silva LADEIRA ${ }^{(a)}$ Adriana Dibo da CRUZ(b) Solange Maria de ALMEIDA(a)}

(a) Universidade Estadual de Campinas UNICAMP, Piracicaba Dental School, Department of Oral Diagnosis, Piracicaba, SP, Brazil.

(b) Universidade Federal Fluminense - UFF, Dental School of Nova Friburgo, Department of Specific Formation, Nova Friburgo, RJ, Brazil.

Declaration of Interests: The authors certify that they have no commercial or associative interest that represents a conflict of interest in connection with the manuscript.

Corresponding Author:

Daniela Brait Silva Ladeira

E-mail: d_brait@hotmail.com

DOI: 10.1590/1807-3107BOR-2015.vol29.0120

Submitted: Dec 17, 2014

Accepted for publication: May 19, 2015

Last revision: Aug 28, 2015

\begin{abstract}
Three-dimensional imaging modalities have been reported to be more accurate than panoramic radiographs (PR) for the assessment of bone components of the temporomandibular joint (TMJ). No exact prior information is available that demonstrates which specific limitations occur in terms of TMJ diagnosis when using PR for this purpose. This study aimed to assess the clinical validity of digital panoramic radiography (DPR) when diagnosing morphological disorders of the TMJ using cone-beam computed tomography (CBCT) images as the gold standard. A sample composed of TMJ images ( $\mathrm{N}=848$ ), including $212 \mathrm{DPR}$ and $212 \mathrm{CBCT}$ images obtained from the same patient, was used to assess any morphological changes in the TMJ. Four appraisers diagnosed all of the DPR images, whereas the CBCT images were used to establish the gold standard. The reliability of each appraiser's response pattern was analyzed using the Kappa test (к), and diagnostic tests were performed to assess each appraiser's performance using a significance level setting of $5 \%(\alpha=0.05)$. Reliability of each appraiser's response pattern compared to the gold standard ranged from a slight-to-moderate agreement $(0.18 \leq \mathrm{\kappa} \leq 0.45)$; and among the different appraisers, the response pattern showed a fair agreement $(0.22 \leq \mathrm{K} \leq 0.39)$. Diagnostic tests showed a wide range among the different possible morphological changes diagnosed. DPR does not have validity when diagnosing morphological changes in the TMJ; it underestimates the radiological findings with higher prevalence, and thus, it cannot be used effectively as a diagnostic tool for bone components within this region.
\end{abstract}

Keywords: Mandibular Condyle; Diagnostic Imaging; Radiography, Panoramic; Cone-Beam Computed Tomography.

\section{Introduction}

The first reports of disorders in temporomandibular joints (TMJ) were cited in the literature in 1918. ${ }^{1}$ Subsequently, diagnostic imaging methods using $\mathrm{X}$-rays have been essential to the morphological assessment of the condyle and glenoid fossa, along with visualization of degenerative signs that go beyond the clinical signs and symptoms of TMJ disorders. ${ }^{2}$

Due to the complexity of the skull base and TMJ components, different types of imaging exams were used for visualization in these regions; three among them include one with a low radiation dose and low cost, commonly 
referred to as panoramic radiography. ${ }^{3}$ Conversely, the use of flat film for TMJ diagnosis is not always sufficient because it requires three-dimensional ratings. ${ }^{4}$ In such cases, tomographic methods have provided excellent data on bone components of the TMJ, despite having disadvantages, such as high cost and high radiation doses. $5,6,7,8,9$

Therefore, cone-beam computed tomography (CBCT) and other tomographic three-dimensional imaging modalities have been reported to be more accurate than panoramic radiographs (PR) for the assessment of bone components of the TMJ and morphological changes with high-resolution quality. $8,9,10,11,12,13$ However, in the literature, there is no exact information that demonstrates which specific limitations occur in terms of TMJ diagnosis when using panoramic images for this purpose. There are several studies ${ }^{14,15,16,17,18}$ designed to inform researchers that panoramic images are valid for assessing specific morphological changes in the TMJ. However, in these cases, the studies are delineated over this specific condition, and they differ in terms of clinical conditions, which is most challenging.

Considering that the panoramic radiograph remains primarily used for the initial evaluation of TMJ, this study aimed to assess the clinical validity of DPR for the diagnosis of morphological disorders of the TMJ using CBCT images as the gold standard.

\section{Methodology}

After this project was approved by the Human Research Ethics Committee of the School of Dentistry of Piracicaba - Universidade Estadual de Campinas - UNICAMP, protocol number 149/2010, 212 medical records were selected from an archive of patients enrolled in clinic dentistry at the authors' public university. These patients accepted the terms of this study by signing an informed consent form. Their medical records contained both exams: a digital panoramic radiograph (DPR) and a CBCT. Furthermore, other inclusion criteria for sample purposes required that patients be at least 18 years of age and no older than age 75, and that they do not constitute any particularly vulnerable group. In addition, exclusion criteria included any exams with low-quality images for TMJ diagnosis.

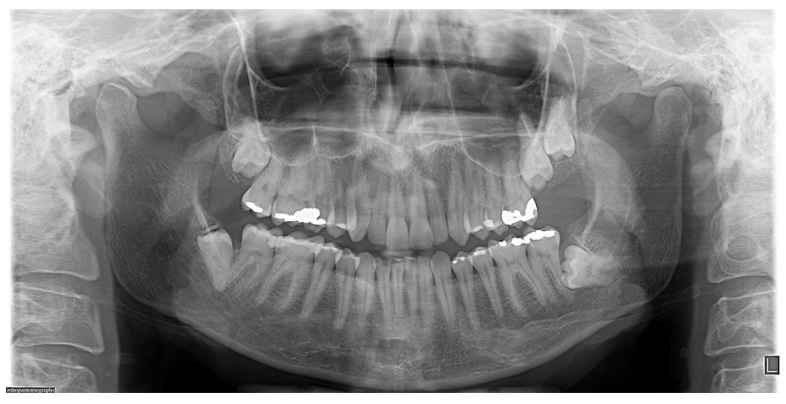

Figure 1. Representative image of a digital panoramic radiograph used in this study.

All DPRs (Figure 1) had been acquired using the same device: a digital panoramic machine, Orthopantomograph OP100 D ${ }^{\circledR}$ (Instrumentarium Corp./Imaging Division, Tuusula, Finland) operating with a filtration of $2.5 \mathrm{~mm}$ aluminum and a focal size of $0.35 \times 0.5 \mathrm{~mm}$, a CCD sensor, and approximately $66 \mathrm{kVp}, 2.5 \mathrm{~mA}$, with an exposure time of $17.6 \mathrm{~s}$. The same occurred for the CBCT images: all had been acquired through a tomograph i-CAT ${ }^{\circledast}$ (Imaging Sciences International, LLC, Hatfield, USA), following the same exam protocol of a field of view (FOV) of $13 \times 17 \mathrm{~cm}, 0.25 \mathrm{~mm}$ voxel size, and approximately 40 seconds of acquisition time at $120 \mathrm{kV}$ and 3-8 $\mathrm{mA}$.

The images for the study were recorded using the specific software related to each device; thus, for DPR, the CliniView ${ }^{\circledR}$ software was used, and for CBCT, the XoranCat ${ }^{\circledR}$ Version 3.1.62 software was used.

All 212 DPRs were made available in blocks $(\mathrm{N}=20)$ to four appraisers with experience providing TMJ diagnosis, in order for them to prepare a radiographical report. Initially, a rehearsal session was held that allowed the appraisers to become familiar with the scoring program related to radiographic reporting and how to appraise the images for this study. To improve their reports, the appraisers could manipulate the images using the software cited. Each TMJ diagnosis was independently made by an appraiser, taking into consideration the right and left sides, respectively. The appraisers viewed the images using the same computer, with a monitor consisting of a 14-inch Liquid Crystal Display (LCD WXGA TFT), while decreasing the luminous intensity of a secluded room. 
To establish the gold standard, two other appraisers with experience providing diagnosis in TMJ independently diagnosed all 212 CBCT exams. Both reports were required to define a unique final diagnostic result. When the responses showed discrepancies, both evaluators needed to come to a proposed consensus answer. The exams were made available in blocks $(\mathrm{N}=10)$ and the appraisers could use all of the software tools cited to improve their reports. In this case, TMJ images of both the right and left side were also individually considered, as illustrated in Figure 2.

The data were tabled, using " 0 " to indicate the absence of morphological changes, and " 1 " to indicate the presence of morphological changes, taking into account any possible marked change as an exclusive diagnosis. Reliability of the response pattern of each appraiser was analyzed using the Kappa test (к). The diagnostic tests (accuracy, specificity, sensitivity, negative predictive value of the [NPV], and positive predictive value [PPV]) were performed to assess performance of the appraisers in diagnosing morphological changes of the TMJ by means of DPRs. All statistical analyses were conducted using a significance level of $5 \%(\alpha=0.05)$.

\section{Results}

Reliability of the response pattern of each appraiser ranged from slight-to-moderate agreement $(0.18 \leq \kappa \leq 0.45)$, with each appraiser's diagnosis being compared against the gold standard. Reliability of the response pattern among the different appraisers, while independently evaluating the diagnosis for each possible morphological change, was considered a fair agreement $(0.22 \leq \kappa \leq 0.39)$.

In relation to possible morphological changes diagnosed by the gold standard, cases of articular loose bodies, hyperplasia, agenesis, ankylosis, and condyle fracture (only in the left TMJ) were not sampled and were considered changes having a low prevalence. The outcomes of the appraisers' performance to these diagnoses are shown in Table 1. Table 2 shows the appraisers' performance to the diagnosis considered as having a medium prevalence in the sample, ranging from $0.47 \%$ to $5 \%$, such as erosion, posterior concavity, bifid condyle, and condylar fracture (of the right side). Finally, cases of flattening, osteophytes, sclerosis, and hypoplasia were considered as having high prevalence in the sample, ranging from $7 \%$ to $80 \%$, and the outcomes of the appraisers' performance to these diagnoses are shown in Table 3.
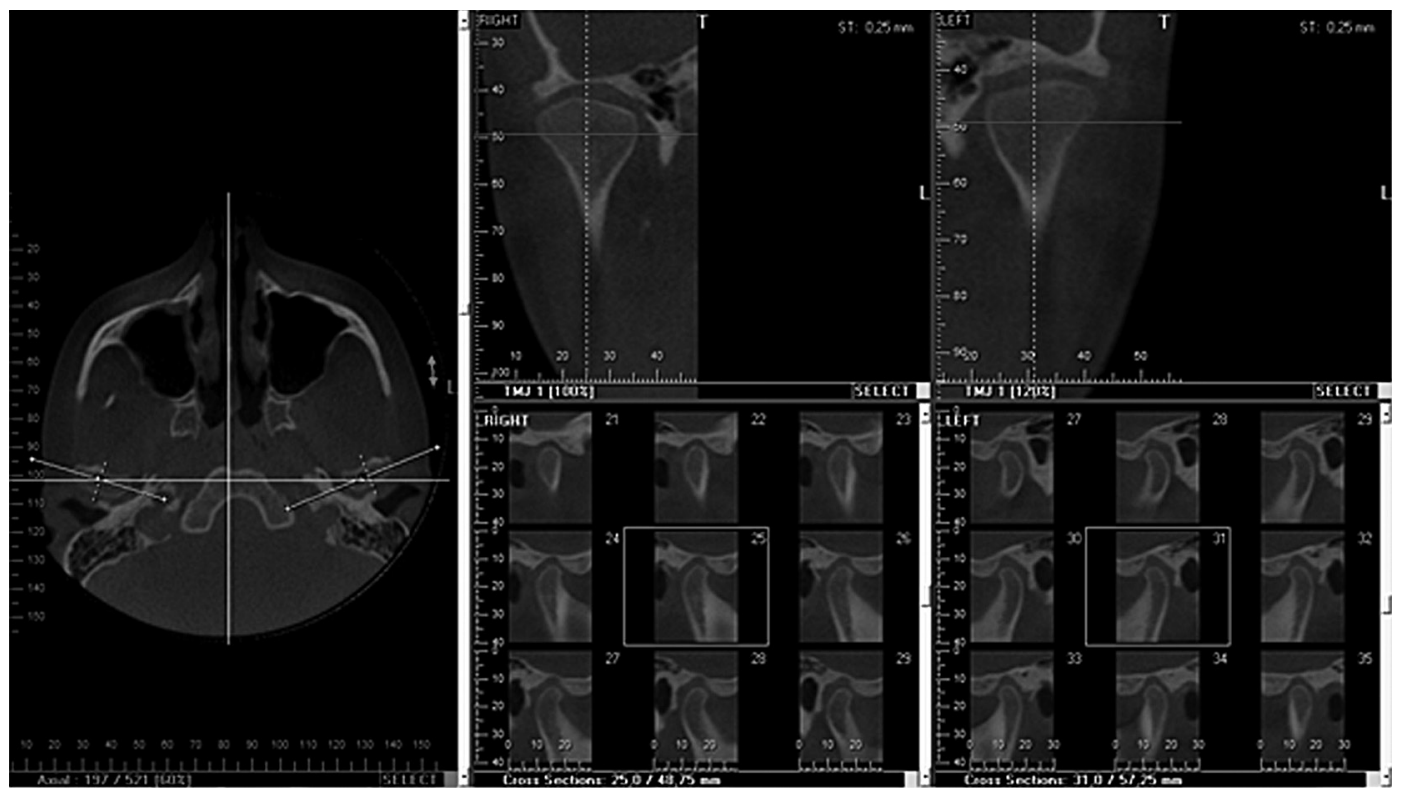

Figure 2. Cone-beam computed tomography images for TMJ diagnosis taking into consideration the right and left sides, respectively. 
Digital panoramic radiography for diagnosis of the temporomandibular joint: CBCT as the gold standard

Table 1. Outcomes of the appraisers' performance to the diagnosis considered as having a low prevalence in the sample.

\begin{tabular}{|c|c|c|c|c|c|c|c|c|c|c|}
\hline \multirow{2}{*}{ Morphological change } & \multirow{2}{*}{$\begin{array}{c}\text { Prevalence } \\
(\%)\end{array}$} & \multicolumn{4}{|c|}{ Frequency (N) } & \multicolumn{5}{|c|}{ Performance measures (\%) } \\
\hline & & $\mathrm{TN}$ & $\mathrm{FN}$ & FP & TP & $A C$ & SE & SP & PPV & NPV \\
\hline Loose body & 0 & 424 & 0 & 0 & 0 & 100 & NA & 100 & NA & 100 \\
\hline Hyperplasia & 0 & 424 & 0 & 0 & 0 & 100 & NA & 100 & NA & 100 \\
\hline Agenesis & 0 & 424 & 0 & 0 & 0 & 100 & NA & 100 & NA & 100 \\
\hline Fracture (Left side) & 0 & 212 & 0 & 0 & 0 & 100 & NA & 100 & NA & 100 \\
\hline Ankylosis & 0 & 424 & 0 & 0 & 0 & 100 & NA & 100 & NA & 100 \\
\hline
\end{tabular}

TN: True negative; FN: False Negative; FP: False Positive; TP: True Positive; AC: accuracy; SE: sensitivity; SP: specificity; PPV: positive predictive value; NPV: negative predictive value; NA: not applicable; Loose body: intraarticular loose bodies (calcified cartilage surrounded by synovial tissue in space articular); Hyperplasia: mandibular condylar hyperplasia (abnormal growth of the condyle); Agenesis: condylar agenesis (absence of all or portions of the TMJ); Fracture: condylar and subcondylar fractures; Ankylosis: immobility or fusion of the joint.

Table 2. Outcomes of the appraisers' performance to the diagnosis considered as having a medium prevalence in the sample.

\begin{tabular}{|c|c|c|c|c|c|c|c|c|c|c|}
\hline \multirow{2}{*}{ Morphological change } & \multirow{2}{*}{$\begin{array}{c}\text { Prevalence } \\
\text { (\%) }\end{array}$} & \multicolumn{4}{|c|}{ Frequency (N) } & \multicolumn{5}{|c|}{ Performance measures (\%) } \\
\hline & & $\mathrm{TN}$ & $\mathrm{FN}$ & FP & $\mathrm{TP}$ & $\mathrm{AC}$ & SE & SP & PPV & NPV \\
\hline Erosion (RS) & 6.60 & 198 & 14 & 0 & 0 & 93.40 & 0 & 100 & NA & 93.40 \\
\hline Erosion (LS) & 4.72 & 202 & 10 & 0 & 0 & 95.28 & 0 & 100 & NA & 95.28 \\
\hline P concavity (RS) & 2.36 & 207 & 5 & 0 & 0 & 97.64 & 0 & 100 & NA & 97.64 \\
\hline P concavity (LS) & 3.77 & 204 & 8 & 0 & 0 & 96.23 & 0 & 100 & NA & 96.23 \\
\hline Bifid (RS) & 1.42 & 209 & 1 & 0 & 2 & 99.53 & 66.67 & 100 & 100 & 99.52 \\
\hline Bifid (LS) & 0.94 & 210 & 2 & 0 & 0 & 99.06 & 0 & 100 & NA & 99.06 \\
\hline Fracture (RS) & 0.47 & 210 & 1 & 1 & 0 & 99.06 & 0 & 99.53 & 0 & 99.53 \\
\hline Cyst (RS) & 1.89 & 205 & 4 & 3 & 0 & 96.70 & 0 & 98.56 & 0 & 98.09 \\
\hline Cyst (LS) & 1.89 & 204 & 4 & 4 & 0 & 96.23 & 0 & 98.08 & 0 & 98.08 \\
\hline
\end{tabular}

TN: True negative; FN: False Negative; FP: False Positive; TP: True Positive; AC: accuracy; SE: sensitivity; SP: specificity; PPV: positive predictive value; NPV: negative predictive value; NA: not applicable; RS: right side; LS: left side; Erosion: local area of rarefaction in the cortical plate of a joint surface, deforming joints; P concavity: posterior concavity (concavity of the posterior surface of the condyle); Bifid: bifid mandibular condyle (duplication of the mandibular condyle); Fracture: condylar and subcondylar fractures; Cyst: Ely's cyst or sub cortical cyst (rounded radiolucent area below the cortical plate or deep in trabecular bone of the condyle).

Table 3. Outcomes of the appraisers' performance to the diagnosis considered as having a high prevalence in the sample.

\begin{tabular}{|c|c|c|c|c|c|c|c|c|c|c|}
\hline \multirow{2}{*}{ Morphological change } & \multirow{2}{*}{$\begin{array}{l}\text { Prevalence } \\
(\%)\end{array}$} & \multicolumn{4}{|c|}{ Frequency $(\mathrm{N})$} & \multicolumn{5}{|c|}{ Performance measures (\%) } \\
\hline & & $\mathrm{TN}$ & $\mathrm{FN}$ & FP & $\mathrm{TP}$ & $A C$ & SE & SP & PPV & NPV \\
\hline Osteophytes (RS) & 30.19 & 148 & 61 & 0 & 3 & 71.23 & 4.69 & 100 & 100 & 70.81 \\
\hline Osteophytes (LS) & 34.91 & 134 & 68 & 4 & 6 & 66.04 & 8.11 & 97.10 & 60 & 66.34 \\
\hline Flattening (RS) & 80.66 & 33 & 111 & 8 & 60 & 43.87 & 35.09 & 80.49 & 88.24 & 22.92 \\
\hline Flattening (LS) & 87.26 & 21 & 123 & 6 & 62 & 39.15 & 33.51 & 77.78 & 91.18 & 14.58 \\
\hline Sclerosis (RS) & 8.02 & 195 & 17 & 0 & 0 & 91.98 & 0 & 100 & NA & 91.98 \\
\hline Sclerosis (LS) & 7.08 & 196 & 15 & 1 & 0 & 92.45 & 0 & 99.49 & 0 & 92.89 \\
\hline Hypoplasia (RS) & 7.55 & 195 & 15 & 1 & 1 & 92.45 & 6.25 & 99.49 & 50 & 92.86 \\
\hline Hypoplasia (LS) & 5.19 & 201 & 10 & 0 & 1 & 95.28 & 9.09 & 100 & 100 & 95.26 \\
\hline
\end{tabular}

TN: True negative; FN: False Negative; FP: False Positive; TP: True Positive; AC: accuracy; SE: sensitivity; SP: specificity; PPV: positive predictive value; NPV: negative predictive value; NA: not applicable; RS: right side; LS: left side; Osteophytes: marginal bone overgrowth on joint surface; Flattening: loss of an even convexity or concavity of the joint out lines; Sclerosis: increased bone density, thickening of the cortical bone on a joint surface; Hypoplasia: Mandibular condylar hypoplasia (underdevelopment or defective formation of the condyle). 


\section{Discussion}

The vast differential from this study in relation to that of previous studies is that the current study did not sample any specific condition of morphological TMJ disorders. The sample used in the present study was randomly selected from a population that showed no further indication or clinical signs and symptoms of TMJ disorders, and thus, any morphological changes of the TMJ would simply be a radiological finding. From a clinical standpoint, the initial detection of morphological changes of the TMJ is often difficult to identify, because an initial clinical diagnosis is routinely based on a suspicion, rather than any visual degenerative signs. Thus, as in many cases, PRs are used to make an initial patient evaluation, along with using the same image to make a TMJ diagnosis.

In accordance with the results of this study, the initial diagnosis of the TMJ using PRs can be problematic due to low reliability of the response pattern of the different appraisers for the presence of morphological changes of the TMJ. The authors of this study also observed a low reliability rate among the different appraisers when diagnosing each possible morphological change. These low rates can indicate a deficiency in the relationship of the panoramic image to its resolution. This could have improved perception to a specific diagnostic, thus improving the intra- and inter-appraisers' outcomes for the same finding. Other studies also found low agreement for diagnosing the TMJ when using panoramic radiography, with values of reliability of the response pattern being approximately $\mathrm{k}=0.31$ in the study by Crow et al. $.^{19} \mathrm{k}=0.19$ in the Schmitter et al. ${ }^{20}$ study; and $\mathrm{k}=0.16$ in the study by Ahmad et al. ${ }^{21}$ thus demonstrating a similar deficiency that was found in the current study. On the other hand, a study by Dahlstrom and Lindvall ${ }^{15}$ reported better values of reliability of the response pattern-approximately $\mathrm{k}=0.50$ - when confronted with the diagnosis from panoramic images against a gold standard. In this case, the improvement in diagnosis can be clarified by the methodology used in the previous study, which was limited to diagnosis of a specific morphological change. When appraisers are well-trained to find a specific diagnosis, it is possible for them to become more susceptible to visualizing that particular diagnosis, which can differ from a clinical condition. ${ }^{14,15}$
Regarding the radiological findings sampled in the current study, which included 424 TMJ evaluations, the authors observed a dissimilar prevalence among the findings. Then, for better contextualization of these findings, the sample was divided into three groups based on the sample prevalence low/medium/high contingent on the prevalence of possible morphological changes diagnosed by the gold standard.

Even though changes in articular loose bodies, hyperplasia, agenesis, ankylosis, and condyle fracture are considered possible morphological changes diagnosed by the gold standard, the authors of this study did not observe them in the current sample. Also, other previous studies ${ }^{17,18}$ did not find cases of hyperplasia and ankylosis in their samples, indicating a low prevalence for these findings in other populations. In relation to the ability of the appraisers to diagnose these possible morphological changes in DPR, the results indicated a perfect diagnosis; however, due to low prevalence in the sample, this can be perceived as a bias. Thus, with this sample, unfortunately, it was not possible to determine a clinical validity of the DPR for these diagnoses.

In terms of other radiological findings, such as erosion, posterior concavity, bifid condyle, and condylar fracture, all of which were considered in the current study to have a medium prevalence (ranging from $0.47 \%$ to $5 \%$ ), each showed great variability of prevalence in previous studies. Only in a study by Pontual et al. ${ }^{18}$ was there a related prevalence similar to that found in the current study. Others studies ${ }^{16,17,22}$ related a wide range of prevalences, ranging from $24 \%$ to $58.5 \%$. Thus, these findings represent possible morphological changes that can be more easily sampled. The appraisers' performance indicated them to be a near-perfect diagnostic for these possible morphological changes in DPR. The decrease in accuracy, specificity, and NPV indicate that there are probabilities of false negatives for these diagnoses; thus, these morphological changes would sometimes be imperceptible to appraisers through a DPR, which was also related in previous studies. ${ }^{23,24}$

The radiological findings of flattening $(80 \%)$, osteophytes (30\%), and sclerosis and hypoplasia (7\%) had the highest prevalence in the current sample. These high prevalences were also found in previous 
studies $9,15,16,18,23,24,25,26,27$ that related prevalence values in their samples for flattening and osteophytes, very similar to the current study. For diagnoses made through DPR, the diagnostic performance decreased more. A vast range of outcome values from the diagnostic test was observed, suggesting that the DPR was not able to detect these radiological findings in the TMJ, because, in a great majority of cases, these changes were imperceptible or, in the worst case, they led to false positive errors. Others studies ${ }^{3,28,29}$ also related that these morphological changes were not perceptible in panoramic radiographs, and similarly, additional studies showed a wide range of outcomes in diagnostic tests. ${ }^{15,24,25}$

One can speculate one of the reasons for the difference in diagnostic performance when assessing bone components of the TMJ when using panoramic radiography. Image formation of the panoramic radiograph follows the principles of linear tomography, with the X-ray beam projecting obliquely upon the long axis of the condyle in a non-parallel way, $3,25,26,27,28,29$ causing overlays and dimensional variations. ${ }^{2,13}$ The medial condylar surface possesses a horizontal rotation to the posterior surface, because the condyle is angled between $15^{\circ}$ and $33^{\circ}$ in a sagittal plane..$^{14}$ In addition, the image can also be affected by the position of the TMJ into an image layer. The image layer of the panoramic machine Orthopantomograph OP $100 \mathrm{D}$ is three-dimensional, with a maximum width of 2.5

\section{References}

1. Pringle JH. Displacement of the mandibular meniscus and its treatment. Br J Surg. 1918;6(23):385-9. doi:10.1002/bjs.1800062307

2. Salemi F, Shokri A, Mortazavi H, Baharvand M. Diagnosis of simulated condylar bone defects using panoramic radiography, spiral tomography and cone-beam computed tomography: a comparison study. J Clin Exp Dent. 2015;7(1):e34-9. doi:10.4317/jced.51736

3. Fallon SD, Fritz GW, Laskin DM. Panoramic imaging of the temporomandibular joint: an experimental study using cadaveric skulls. J Oral Maxillofac Surg. 2006;64(2):223-9. doi:10.1016/j.joms.2005.10.035.

4. Zhang ZL, Cheng JG, Li G, Shi XQ, Zhang JZ, Zhang $\mathrm{ZY}$ et al. Detection accuracy of condylar bony defects in Promax 3D cone beam CT images scanned with different $\mathrm{cm}$ for this area, and it is approximately $1 \mathrm{~cm}$ wide in the central portion, where the image magnification is uniform. ${ }^{11}$ In cases where the condyle possesses an on-average width of $2.0 \mathrm{~cm}$ and a length of $1.0 \mathrm{~cm},{ }_{1}^{14}$ its dimensions are compatible with the width of the layer image; however, only one part becomes positioned in the central portion of this layer. For that reason, it is important to note that the TMJ image in panoramic radiography will always result in distortions. Thus, the authors of this study strongly advise against use of panoramic radiography to diagnose these studied morphological changes, due to the vast majority of these findings were imperceptible; however, sometimes distortion of the TMJ image can induce a false positive diagnosis, which can lead to further investigations being required. Consequently, prescription of the image exam using high-resolution quality, such as tomography to assess bone components of the TMJ and morphological changes, should be done exclusively based on the clinical signs and symptoms of TMJ disorders.

\section{Conclusion}

According to the results of this study, DPR does not have validity for diagnostic or morphological changes in the TMJ, because it underestimates the radiological findings with higher prevalence; thus it cannot be used effectively as a diagnostic tool for bone components in this region.

protocols. Dentomaxillofac Radiol. 2013;42(5):20120241. doi:10.1259/dmfr.20120241

5. Spin-Neto R, Matzen LH, Schropp L, Gotfredsen E, Wenzel A. Factors affecting patient movement and re-exposure in cone beam computed tomography examination. Oral Surg Oral Med Oral Pathol Oral Radiol. 2015;119(5):572-8. doi:10.1016/j.oooo.2015.01.011

6. Endo M, Terajima M, Goto TK, Tokumori K, Takahashi I. Three-dimensional analysis of the temporomandibular joint and fossa-condyle relationship. Orthodontics (Chic). 2011;12(3):210-21.

7. Yadav S, Palo L, Mahdian M, Upadhyay M, Tadinada A. Diagnostic accuracy of 2 cone-beam computed tomography protocols for detecting arthritic changes in temporomandibular joints. Am J Orthod Dentofacial Orthop. 2015;147(3):339-44. doi:10.1016/j.ajodo.2014.11.017 
8. Zhang ZL, Shi XQ, Ma XC, Li G. Detection accuracy of condylar defects in cone beam CT images scanned with different resolutions and units. Dentomaxillofac Radiol. 2014;43(3):20130414. doi:10.1259/dmfr.20130414

9. Alkhader M, Ohbayashi N, Tetsumura A, Nakamura S, Okochi $\mathrm{K}$, Momin MA et al. Diagnostic performance of magnetic resonance imaging for detecting osseous abnormalities of the temporomandibular joint and its correlation with cone beam computed tomography. Dentomaxillofac Radiol. 2010;39(5):270-6. doi:10.1259/dmfr/25151578

10. Beloor Vasudeva S, Kameko N, Endo A, Okano T. Influence of horizontal condylar angle and $\mathrm{x}$-ray projection angle on the appearance of the condyle on lateral temporomandibular joint panoramic radiographs. Oral Health Dent Manag. 2012;11(4):177-84.

11. Ladeira DB, Cruz AD, Almeida SM, Bóscolo FN. Influence of the intergonial distance on image distortion in panoramic radiographs. Dentomaxillofac Radiol. 2012;41(5):417-21. doi:10.1259/dmfr/59761876

12. Patel A, Tee BC, Fields H, Jones E, Chaudhry J, Sun Z. Evaluation of conebeam computed tomography in the diagnosis of simulated small osseous defects in the mandibular condyle. Am J Orthod Dentofacial Orthop 2014;145(2):143-56. doi:10.1016/j.ajodo.2013.10.014

13. Honey OB, Scarfe WC, Hilgers MJ, Klueber K, Silveira AM, Haskell BS et al. Accuracy of cone-beam computed tomography imaging of the temporomandibular joint: comparisons with panoramic radiology and linear tomography. Am J Orthod Dentofacial Orthop 2007;132(4):429-38. doi:10.1016/j.ajodo.2005.10.032

14. Farman AG, Ludlow JB, Davies KL, Tyndall DA. Temporomandibular joint imaging: a comparative study of diagnostic accuracy for the detection of bone change with biplanar multidirectional tomography and panoramic images. Oral Surg Oral Med Oral Pathol Oral Radiol Endod 1995;80(6):735-43. doi:10.1016/S1079-2104(05)80259-8

15. Dahlstrom L, Lindvall AM. Assessment of temporomandibular joint disease by panoramic radiography: reliability and validity in relation to tomography. Dentomaxillofac Radiol 1996;25(4):197-201. doi:10.1259/dmfr.25.4.9084273

16. Hintze $H$, Wiese $M$, Wenzel A. Cone beam CT and conventional tomography for the detection of morphological temporomandibular joint changes. Dentomaxillofac Radiol. 2007;36(4):192-7. doi:10.1259/dmfr/25523853

17. Nah KS. Condylar bony changes in patients with temporomandibular disorders: a CBCT study. Imaging Sci Dent. 2012;42(4):249-53. doi:10.5624/isd.2012.42.4.249

18. Pontual MLA, Freire JS, Barbosa JM, Frazão MA, Pontual AA, Silveira MMF. Evaluation of bone changes in the temporomandibularjoint using cone beam CT. Dentomaxillofac Radiol. 2012;41(1):24-9. doi:10.1259/dmfr/17815139
19. Crow HC, Parks E, Campbell JH, Stucki DS, Daggy J. The utility of panoramic radiography in temporomandibular joint assessment. Dentomaxillofac Radiol. 2005;34(2):91-5. doi:10.1259/dmfr/24863557

20. Schmitter M, Gabbert O, Ohlmann B, Hassel A, Wolff D, Rammelsberg P et al. Assessment of the reliability and validity of panoramic imaging for assessment of mandibular condyle morphology using both MRI and clinical examination as the gold standard. Oral Surg Oral Med Oral Pathol Oral Radiol Endod. 2006;102(2):22-4. doi:10.1016/j.tripleo.2005.07.039

21. Ahmad M, Hollender L, Anderson Q, Kartha K, Ohrbach $\mathrm{R}$, Truelove EL et al. Research diagnostic criteria for temporomandibular disorders (RDC/TMD): development of image analysis criteria and examiner reliability for image analysis. Oral Surg Oral Med Oral Pathol Oral Radiol Endod. 2009;107(6):844-860. doi:10.1016/j.tripleo.2009.02.023

22. Barghan S, Merrill R, Tetradis S. Cone beam computed tomography imaging in the evaluation of the temporomandibular joint. J Calif Dent Assoc. 2010;38(1):33-9.

23. Hintze H, Wiese M, Wenzel A. Comparison of three radiographic methods for detection of morphological temporomandibular joint changes: panoramic, scanographic and tomographic examination. Dentomaxillofac Radiol. 2009;38(3):134-40. doi:10.1259/dmfr/31066378

24. Masood F, Katz JO, Hardman PK, Glaros AG, Spencer P. Comparison of panoramic radiography and panoramic digital subtraction radiography in the detection of simulated osteophytic lesions of the mandibular condyle. Oral Surg Oral Med Oral Pathol Oral Radiol Endod. 2002;93(5):626-31. doi:10.1067/moe.2002.121704

25. Güler N, Yatmaz PI, Ataoglu H, Emlik D, Uckan S. Temporomandibular internal derangement: correlation of MRI findings with clinical symptoms of pain and joint sounds in patients with bruxing behaviour. Dentomaxillofac Radiol. 2003;32(5):304-10. doi:10.1259/dmfr/24534480

26. Alexiou K, Stamatakis H, Tsiklakis K. Evaluation of the severity of temporomandibular joint osteoarthritic changes related to age using cone beam computed tomography. Dentomaxillofac Radiol. 2009;38(3):141-7. doi:10.1259/dmfr/59263880

27. Ruf S, Pancherz H. Is orthopantomography reliable for TMJ diagnosis? An experimental study on a dry skull. J Orofac Pain. 1995;9(4):365-74.

28. Winocur E, Reiter S, Krichmer M, Kaffe I. Classifying degenerative joint disease by the RDC/TMD and by panoramic imaging: a retrospective analysis. J Oral Rehabil. 2010;37(3):171-7. doi:10.1111/j.1365-2842.2009.02035.x

29. Roberts D, Pettigrew J, Ram C, Joseph PM. Radiologic techniques used to evaluate the temporomandibular joint; I. Conventional methods. Anesth Prog. 1984;31(5):197-206. 